\title{
Preserving Privacy Techniques for Autonomous Vehicles
}

\author{
Sravanthi Keesara ${ }^{1}$, Vijay Kumar Burugari ${ }^{2}$, Prabha Selvaraj ${ }^{3}$, Kanmani $\mathbf{P}^{4}$ \\ Amit Kumar Tyagi ${ }^{5}$ \\ ${ }^{1}$ VNR Vignana Jyothi institute of Engineering and Technology, India, sravanthireddy.k8@ gmail.com. \\ ${ }^{2}$ Koneru Lakshmaiah Education Foundation, India, vijaybru@gmail.com \\ ${ }^{3}$ VIT-AP University, India, Email prabha.dw@ gmail.com \\ ${ }^{4}$ K.S.Rangasamy College of Technology, India, pkanmaniit@ gmail.com \\ ${ }^{5}$ Vellore Institute of Technology, India,amitkrtyagi025@gmail.com
}

\begin{abstract}
In this automated world, there will be increase in population, more city development and less space for parking results in parking related problems. In our day-to-day life vehicle became an important resource. To go anyplace we require a vehicle, when we are using vehicle, parking will be the big problem. Due to insufficient parking lots and no knowledge about the parking lots (i.e., do not the parking lots places). To park a vehicle manually we have to wait in line to take the token for parking which is a time-consuming process. There is a need to find a secured, smart, efficient system for finding nearest unoccupied parking lots, to reserve parking lot, guidance to navigate to parking lot, to negotiate parking fee and finally to secure the vehicle in parking places. Smart parking system is introduced by Intelligent Transport Systems (ITS). This article gives an overview on different smart parking services like reserving parking lots, navigating to parking lots, finding nearest parking lots, how to provide security to vehicle. It shows the cons and pros of every smart parking system.
\end{abstract}

Key words: Privacy, Autonomous Vehicles, parking, Security, Block chain Technology

\section{INTRODUCTION}

In this digital world privacy is entirely lacking. If a bank is considered where it is an Open Banking and if you have an account, the complete transaction is gathered and the information is sold to insurance companies and credit agencies These people use the available details to make decision whether worth to advertise or not. This is a big issue of financial privacy is a big issue today where many companies call to customers' irrelevantly. A new horizon is going to arise in near future with digital freedom by the use of Block chain technology. Block chain [1] is a public ledger which is decentralized and distributed. Every Block in it is made up of digital pieces of information. When a Bitcoin Block chain is considered, when someone try to identify the user information and the transactions id, it is denied. Even though transactions on the Block chain are not completely anonymous, users personal information is limited to their digital signature or username. So it provides security and trust in many ways. of the many examples where Block chain can help is in Travel Insurance, i.e., delays and cancellation of flights can be prevented by Travel Agencies using Block chain. Block chain, which was just a virtual ledger, is spreading like a wave across extensive number of industries. Businesses are expected to spend colossal amount of money on this powerful technology in this year. From Insurance to gaming to cannabis, almost all industries are looking to develop and apply Block chain Technology, there is hardly any domain not looking for Block chain applications because this technology is more likely to transform the industries. There are several existing systems available for flexible parking managements which are included here as:

In Intelligent parking systems - they made everything online i.e., reservation and payments for reserving parking lots. Online decision to accept or reject new driver request (refer figure 1).

A reservation-based smart parking system-According to the number of parking lots the price is going to change dynamically. So, driver has to think about budget.

Parking Space Reservation based on VANETs-provides road side unit to collect traffic information accordingly provides parking lot reservation.

Use either SI (MKS) or CGS as primary units. (SI units are strongly encouraged.) English units may be used as secondary

\section{RELATED WORK}

The idea behind Block chain technology was started in 1991 by research scientists Stuart Haber and W. Scott stornetta [2] where they applied a computationally practical solution so that it cannot be tampered backed up by time-stamping digital and secured the block chain by using cryptography. In 1992, several certificate documents were integrated into a block by using Merkle trees but the licence for this technology 
was cancelled in 2004. Hal Finney, a computer scientist and cryptographic activist introduced a system called RPoW (Reusable Proof of Work) in 2004. Reusable Proof of Work (RPoW) receives a non-exchangeable hash cash-based proof of work token and it generates RSA-signed token that could then be transferred from person to person. RPoW stores the registered tokens on trusted server to avoid double spending problem and it allows the people overall the world to verify the correctness and integrity in real time.it was the first step in the history of bitcoin (crypto currencies).

In 2008, Satoshi Nakamoto is the inventor of Bitcoin. It is a crypto currency and it is known as decentralized peer-peer electronic cash system. In cryptography mail list it was posted by Satoshi Nakamoto. It works based on hash cash proof of work algorithm. Instead of using Reusable Proof of Work (RPoW), to avoid double spending problem, a decentralized peer-to-peer protocol provided by bitcoin can be used for verifying the correctness of all transactions. Individual miners mined Bitcoins for a reward using the proof-of-work system and then verified by the network`s decentralized nodes. Bitcoin came into existence on January $3^{\text {rd }}, 2009$, when Satoshi Nakamoto mined the first bitcoin block, which had a reward of 50 bitcoins. In the world, the first bitcoin transaction is done on January $12^{\text {th }}, 2009$. Santoshi Nakamoto is the first sender of bit coin, he transferred 10 bitcoins to the Hal Finney (first receipt of bitcoins). The first application of Block chain is bitcoin. For the first time, it is possible to transfer money without any trusted third party. In 2013, Vitalik Buterin, bitcoin Magazine co-founder and programmer stated that for building decentralized applications, bitcoin needed a scripting language. This statement is not agreed in the community. Blockchain-based distributed computing platform, Ethereum (global, open-source platform) is developed by Vitalik started developing which had scripting features called smart contracts. Smart contracts used for exchanging shares, property documents or anything of value among unknown people in conflict free transparent manner. It avoids trusted third party (services of middleman). Smart contracts are the executable logic that runs on Block chain network. Key properties of smart contracts: Autonomous (the creator doesn't have to participate after deploying contract), Decentralized (it does not have central server). The first application of smart contracts electronic transactions was proposed by nick Szabo. In particular programming languages smart contracts are written, compiled and converted into byte code by Ethereum Virtual Machine (EVM). Applications are run inside Ethereum Block chain and they are called as DApps(Decentralized Applications) [3]. For example, gambling, financial exchanges. Ether is the crypto currency of Ethereum. it can be used to pay online payments when executing smart contracts. Ether can be transferred between accounts. Bhandari et. al. gives a view about the scheduling techniques with energy efficiency and explains a technique for transmission of packet enhancing the AODV routing method [20]. Saibaba describes an efficient and cost effective surveillance technique [21]. Virtualization in cloud environment at IaaS is explained by Amudhavel [22]. Pradhan et. al. provides an adaptive PVD technique with high hiding capacity and minimum distortion [23]. Rao K. T. et. al. discussed a modern method for securing the transaction of data in private cloud [24]. Rambabu et. al. has presented a review on contemporary models and explained the necessity for intrusion detection [25].A survey on the challenges and issues in security in WSN is described by Shaik et. al. [26]. Knox'ified Hadoop cluster design in a new method is said by Sirisha et. al. [27]. Raghava Rao et. al. discussed a technique to transfer traffic information to the vehicle [28]. Anil Kumar explains a technique to monitor driver fatigue to prevent vehicle accident [29]. Joshua Alvaro Fitzhan explains the challenges of smart contracts and how it is used in Indonesian industries [30]. Author discusses how digital legislation can be made for recommendations on making amendments to the current civil using smart contract.

\section{IMPORTANCE AND SCOPE OF BLOCKCHAIN TECHNOLOGY - TODAY AND IN NEAR FUTURE (APPLICATION -WISE)}

Block chain is a list of blocks linked with each other using cryptography. it is having distributed ledger, stored all the transaction in secured manner and it is transparent to avoid security attacks. Block chain is the fastest growing technology in IT sector, financial sector. Around $74 \%$ of organizations that have adopted Block chain globally, and this number will be increased day by day. Block chain main aim is to provide security for transactions. Many organizations are started adopting Blockchain technology because of its benefits to the industry today. Not only in financial sector, in all other sectors, it gained lot of attention. Blockchain technology is used for secure transfer of money, property without Trusted Third Party (TTP).

\section{Some Importance of Block chain are listed in [4] and here} as:

Security purposes: The digital world is full of hacker's who looks to breach or stole the private data. With the Block chain technology, the data is stored in a robust manner. Hacking or modifying the stored data in Block chain is impossible.

Less expensive: In market, financial models are more expensive to implement. But, with Block chain it is inexpensive. Faster transaction: to implement transactions over Block chain network it takes less time.

Transparency: Block chain contains a distributed ledger which permanently records all the transactions and it is transparent (anyone can verify the records on ledger from anywhere).

Financial efficiency: Block chain technology allows one to perform transaction without involvement of trusted third party. For example, Banks. Protect business from fraud: 
Block chain is an open source ledger where all the transactions are transparent. So, it is very easy to find fraud.

Removes intermediators fees: Block chain avoids trusted third party. So, no intermediate fees required. High level of reliability: Block chain has high level of reliability with its open source ledger.

Scope of Blockchain [5]: Block chain technology has a great future in financial and non-financial fields. The financial organisations are not able to handle the workload after demonetization and thus problems are raised by having centralized system for handling financial transactions. RBI took a decision to have digitization in banking sector. Block chain technology with financial transactions give lot of benefits, such as avoiding modifications in financial markets, transactions are processed very fast, money will be saved like no trusted third party here, drastic reduction in time for validating transactions, it ensures tight security, protects from cyber-attacks. Block chain reduced black-money dealings because each and every transaction are stored permanently in blocks.

Block chain technology has great future in many other areas other than the financial field.

Digital Advertising: Digital advertising faces lot of challenges. Such as, lack of transparency, domain fraud, long payment modules. Transparency is provided by Block chain to the supply chain as it fetches trust in a trust less environment by blocking the bad players in supply chain.

Cyber Security: Block chain is a public ledger (all the transactions are stored permanently; this information is transparent)

Voting: with the use of Block chain, voting irregularities could be minimized.

Healthcare: managing patient records, doctors can easily track patients' details (like previous diseases).

In major cities in crowded areas at peak hours' traffic congestion problem arises, due to unavailable of parking lots for reservation. To reduce this type of problems intelligent reservation systems proposed urban parking systems. This system can identify and process the information about parking lots to drivers any time. Even though it is not able to reserve and guide about parking lots to each of drivers.

\section{SERVICES AND FEATURE PROVIDED}

\section{SERVICE PROVIDED}

This article mainly focuses on services provided by smart parking systems like reserving in online a parking lot for vehicle, finding nearest parking lot and it also discusses about fuzzy logic-based systems with parking features for self-driving cars i.e., parallel parking, perpendicular parking of a car, Detection and monitoring of the parking facility. Users can Bargain parking fee, parking guidance and route negotiation (shortest path) etc. Consortium Block chain-to store parking lot offers and reservation of parking lots. Note that Data integrity, Decentralized system, and providing cryptography are best feature/ functionality of Block chain technology.

\section{FEATURE}

The information about budgets of different parking systems and features provided by various parking system like less cost implementation with reduced power consumption, real time location-based information and guidance towards destination and better results for car searching in large parking lots. This article explains about Fuzzy logic-based systems, Vision-based systems, Agent-based systems, Wireless sense-based systems, GPS-based systems, Vehicle communication systems. Features provided by these systems are Solves complex traffic environment, dynamic distribution, preserves vehicle driver's information secure with low communication and computation overheads and provides efficient reservation of parking lot. finally, provides privacy to the owner of vehicle while using smart parking reservation system (no third trusted party).

- Detecting parking lot

- Availability of parking lot

- Collection of parking charges.

- Predicting traffic flow.

- Declining searching path.

- Finding average walking time and travel time.

\section{MOTIVATION}

Now-a-days finding parking lots become more complex. Instead of searching parking lots manually, this generation people are using smart applications. Users are reserving parking lots in online to manage time efficiently. To utilize online services, users have to enter all his private information into the application. All the private information should be kept more secure from outsiders if not they may mislead this information for their own profits. In this article we discussed the different ways to secure our parking information and existing technologies to have smart parking system. This section discusses how much important the smart parking systems in or day-to-day life. Now, next section discusses a Use Case model for smart parking system.

\section{THREAT MODEL: A USE CASE [6]}

In intelligent parking system, vehicle drivers need to register by giving their private information in the application in order to use all the services provided by parking owners. In case of fraud application there is chance to misuse the driver private information like selling vehicle information and driver information to the ad agencies. To reserve the nearest parking lots, vehicle driver has to give permission to track the vehicle. So, there is a chance of jamming attack through road-side units. The network will jam by attackers with transmitters that are stronger than embedded sensors. This attack prevents the receipt of sensed data from the WSN gateway, preventing 
the transfer of data on parking lot status to the RSU. Therefore, the RSU cannot instructor schedule vehicles in the community where parking lots are currently required. Hence, this section discusses the various attacks on smart parking system. Now, next section discusses about the existing systems in intelligent parking system.

\section{EXISTING SOLUTIONS}

Sabbir Ahmed et al. proposed an integrated smart parking system using block chain [7]. In this system, there are 3 modules

- user

- parking service provider

- Block chain network

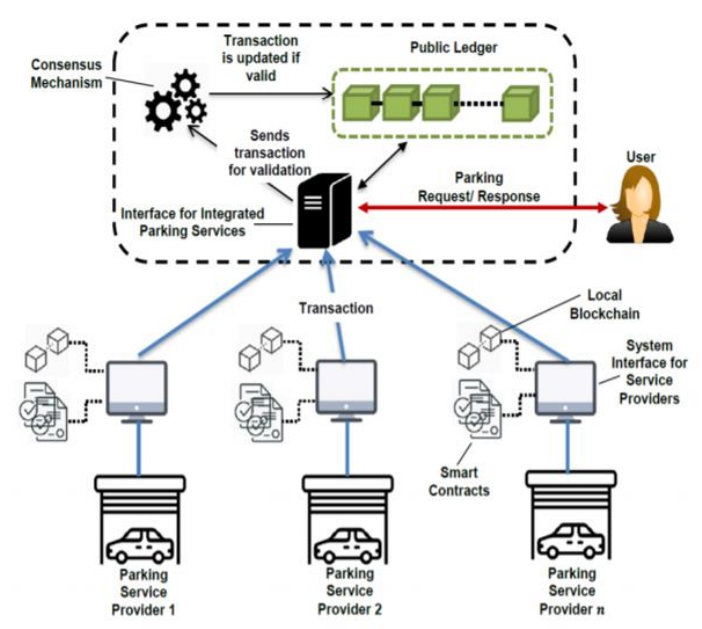

Figure 1: Integrated Smart Parking System overview

Blockchain network consists of public ledger (displays all transaction to public) and it updates with valid transactions. A consensus mechanism is there to verify transactions. User has to request reservation of parking lot. The architecture shows the integrated smart parking system. For example, each parking service provider gives single parking area, whenever a driver requests for the nearest parking lot, the system checks all parking provider's area, and which is nearer that will be assigned to driver. While confirming the parking lot the transaction is noted on the local ledger of parking provider as well as public ledger after validating the transaction.

It provides separate application to interface each of users (vehicle owner) for interacting with it. Every parking lot has a local ledger (local block). Here, two types of transaction systems are there. First one is data produced by sensor at parking lot. It generates the availability of parking lot as transaction. The provider has smart contract to generate a transaction if parking lot is changed from "vacant" to "occupied". The transaction is generated by sensor send to local ledger then consensus Block chain verifies the transaction and then updates in public ledger. It uses Smart contracts for parking fares. Service providers decide parking fares according to time and sent to Block chain. When there is change in price is dynamically updates in Block chain public ledger and Parallel local ledger updates. The user downloads a smart parking app and sends a request for nearest parking lot reservation then service provider checks the user data and book a slot for user. Here there is no third trust party.

A Smart Hybrid System for Parking Space Reservation [8] in VANET-proposed a centralized management of parking in the city to solve traffic congestion and reservation of parking space problems as said by Farhad Rad et. al. Here, they used adoptive neuro-fuzzy system (ANFIS) and multi-objective genetic algorithm to overcome above problems.

ANFIS: This method collects the traffic flow information from VANET (Vehicle Ad-Hoc Network) and predicts the traffic flow using neural networks. it combines all traffic information to predict accurate traffic flow. so that we can estimate the travelling time of a driver to reach parking lot.

Multi-Objective Genetic Algorithm: It processes all the parking requests in period of time simultaneously and generates short path parking lots to all applicants at same period of time. So that time-consuming and cost of parking lot are minimized.

Fitness function: This function is in multi-objective genetic algorithm; it models parking reservation system. Here driver can select cheap parking lots and nearest parking lots. By this function, nearest parking lot can be selected by the driver so that we can reduce walking time.

Crossover: It checks number of parking lots allotted to a particular vehicle driver so that the capacity does not exceeds.

Mutation: random mutation operator is used. Randomly allocates parking numbers.

Simulation: Here the collected data is classified into time series (time intervals of traffic flow). For training proposed system ANFIS this data is used. it predicts accurate traffic flow for present.

Wesam Al Amiri et al. described Private Information retrieval-to secure driver information [9]. Here, system initialization is done by offline trusted authority (It registers parking drivers and provides the secret keys to access the parking lot offers in Block chain and creates public key certificates for parking lot owners to write parking offers on consortium Block chain.). Consortium Block chain network provides decentralized parking lot services and allows only authorized parking lot owners to submit their parking offers. it stores all the parking transaction details on shared ledger using pre-defined consensus algorithm. Every parking lot consist an IoT device which gives information about parking lot availability, i.e., occupied or free. Now parking drivers can retrieve parking offers for reservation which are nearer to them on consortium Block chain without providing private information with the use of Private Information Retrieval (PIR) technique (It secures drivers information from parking lot owners) using their smart phones and they can make online payment for parking lot reservation. 
Faheem et al. discussed about the importance of intelligent parking system [10]. Here, intelligent parking system is a part of Intelligent Transportation System (ITS). It provides a survey on different intelligent parking services used for guiding user about parking lots, information about available parking lots (number of parking lots) management of parking facility. Intelligent parking services are designed with new functionalities which manages the parking facility. In future the intelligent parking system provides the following services:

- Parking reservation system and availability of parking information system should provide advanced tracking services. (i.e., system should provide the availability of parking lots to each driver before they enter).

- Make parking service charges digital currency.

- For safe driving there should be an automated navigation assistance system.

- To achieve best possible traffic management there should be in built navigation system.

- Provide security measures for car safety.

For better results even we can add the parking payment using smart card so that the driver can quickly easily access the parking lot. so that we can avoid the man power at system. This system will decrease the traffic at streets.

The parking service provider provides a parking lot and updates availability of parking lots and price of parking lot in integrated system.

\section{DIFFERENT TECHNOLOGIES}

\section{i. AGENT BASED INTELLIGENT PARKING}

Multi-agent system is a modeling technique. Agent-Based Intelligent Parking Negotiation and Guidance System(ABIPNGS)(In agent-based parking assistance system we are having agent environment(it consists of multi-agents)). All the car parking providers interact with car park agents, in the same way drivers will interact with driver agent. Utility agent is for giving seller/buyer information. Rout guidance agent is for showing the shortest path to parking lots. Now driver will request for parking to driver agent. There will a parking request and negotiation about parking fee between driver agent and car park agent. In this system driver doesn't approach directly to the parking service provider, there are agents in between the parking providers and vehicle drivers. The driver agent will be connected with route guidance agent to find the route for parking. Driver agent is used to send parking request to the parking agents and to negotiate with parking agents. Driver agent finds the cheaper and nearer parking lot and reserves that parking lot for driver to park car, this complete explanation is provided in figure 2 . The driver can check for cheaper and near parking lots.

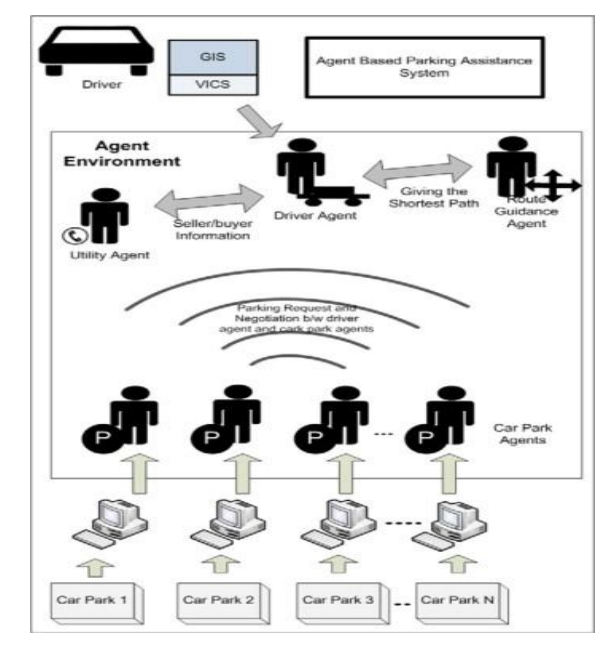

Figure 2: Parking support system based on agent.

\section{ii. FUZZY-LOGIC BASED PARKING SYSTEM}

This model utilizes a self-training system that benefits from human abilities to automatically get a functioning car control system. The fuzzy system has been implemented in the artificial neural architectural design. It is why training data are retrieved, generalized and adopted. Ultrasound and 3D vision sensors can be used to identify parallel parking spaces. Ultrasonic sensor identifies objects and obstacles longitudinally.

\section{iii. WIRELESS SENSOR BASED}

This system is composed of wireless sensors installed in a parking lot. Every parking lot contains a sensor node to detect whether parking lot is free or not. Every node has light, sound and hearing capability sensor for wireless sensor network-based car parking management system. Wide area architecture, distributed accessing methods allow the users to get information about the parking lots on their ways. This system uses motion detectors, cameras and microphones for detecting auto mobiles. All the data in sensors are processed in WSN, and then posted into web. Users can access the data from internet.

Disadvantages: Usage of video camera is very expensive. And it generates huge data which is difficult to transmit over wireless network.

Electromagnetic sensors are linked via an electricity distribution network and data sensing records. Figure 3 illustrates wireless sensor network with magnetic sensors. Here the magnetic sensors are used in nodes of parking lots. Once driver enters into the parking area, vehicle is directed to parking lot using route guidance system. These sensors sense the disturbance in the earth magnetic field for identifying the vehicle. 


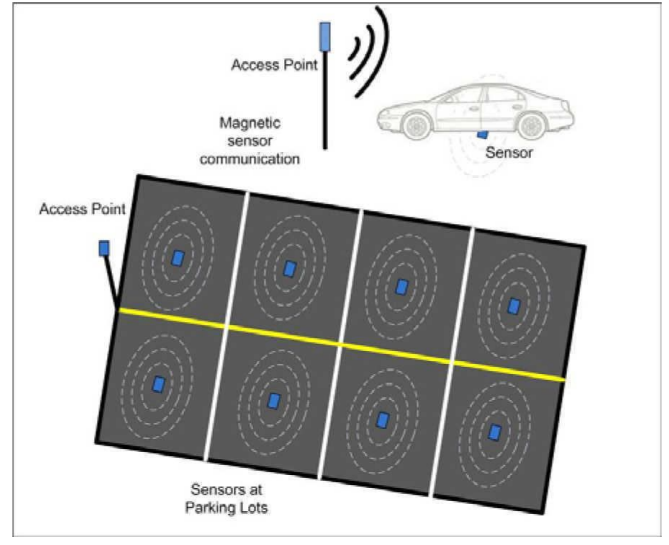

Figure 3: Magnetic sensor inWSN system

\section{iv. GPS BASED SYSTEMS}

\section{SERVICE PROVIDING}

It offers location information and parking facilities available with the help of GPS identifies the location of parking lots near the destination to the drivers. Cerreo mainly focused on-street parking such as storage of parking information and p2p exchange. Arnott and rowse modeled parking system without considering traffic congestion into consideration Chon et al. developed a location based system called NAPA (Nearest Available Parking Lot Application). It helps to find location of parking lot in places like airports, but there is no information about availability of free parking lots.

\section{v. VEHICULAR COMMUNICATION SYSTEMS}

\section{FEATURE}

Provision of distribution of parking data for mobile vehicles.

\section{SERVICE PROVIDED (SPARK)}

- Navigation on real-time car park

- Protection against smart theft (all vehicles are secured by

RSUs, if any car moves illegally from the parking lot it can be detected by RSUs).

- Drivers will get all the information regarding parking lots.

Using VANET communication technology, in figure 4, SPARK is described by using RSUs at parking lot to monitor and manage the entire parking facility. It is used to find criminal activities on vehicles. (for example, anyone is trying to steal the car, that will be noticed by RSU, and this sort of issues can be solved by RSU).

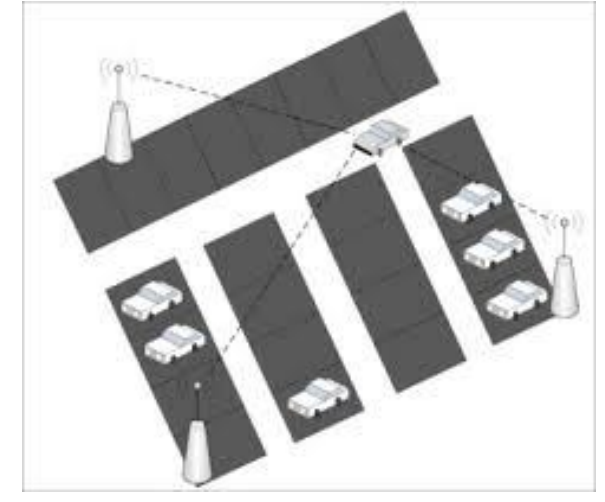

Figure 4: Vehicular communication-based smart parking technique

\section{vi. VISION BASED SYSTEMS}

By using ultrasonic sensor and short range radars(SRR) we can achieve parallel parking by finding free parking lot.Due to the high price on laser scanner, the vision-based technology is most widely used in perpendicular parking. Ultrasonic, SRR are not appropriate to find free slopes. Visually unsupervised vision-based system for the detection of availability of the parking lot for drivers to monitor continuous parking operations. This technique has three main processing:

- Pre-processing of the camera-captured raw pictures.

- The image shadows are removed to avoid distortion.

- Communication between stationary cameras and visible parking lots considered as conclusions.

A smart car search technology has been installed to identify vision-related car information like car colour, the type of license plate being used (because storing license numbers is unreliable). This is done using the cameras on the roads. This system is mainly used in outdoor car parking areas.

Real time car parking is used to deliver the number of cars parked and parking lots free of charge through image processing here. This program uses pictures for vehicle identification. A camera is used when the car is approaching the car park to catch the reference image. Photo series is taken and with the aid of the prewitt edge identification technique compared to parked car photos.

Kyoungwook Min et. al. enables parking vehicles without human interaction. Here vehicle steering is controlled to park at parking lot [11]. For autonomous vehicle parking, they used two turning radius circles to find steering value of vehicle about parallel and perpendicular parking method. These two cars turning radius circles are initially minimal radius circles and are updated as a parking lot location in parking mode in real time. This control system provides automatic parking service in parking area with limited speed. Automated Vehicle follows route generated by AVP system with limited speed in parking lots. Its main feature is to park at parking lot as backward/forward parallel and perpendicular parking method. 
Figure 5, in this model application layer-through which driver can retrieve parking lot information and can reserve parking lot which is nearer to location. Omar Ahmed Abdulkader explained a system [12] provides real time information about

- Availability

- Reservation

- Online payment solutions

- Reducing Traffic congestion.

So that driver can reduce traffic congestion and driving time to reach parking lot. Middleware layer -all the parking lot information is stored in cloud. Network layer-collects all the parking lot availability information from the sensors, RFID to update parking status in middleware and application layer which is provided to the drivers.

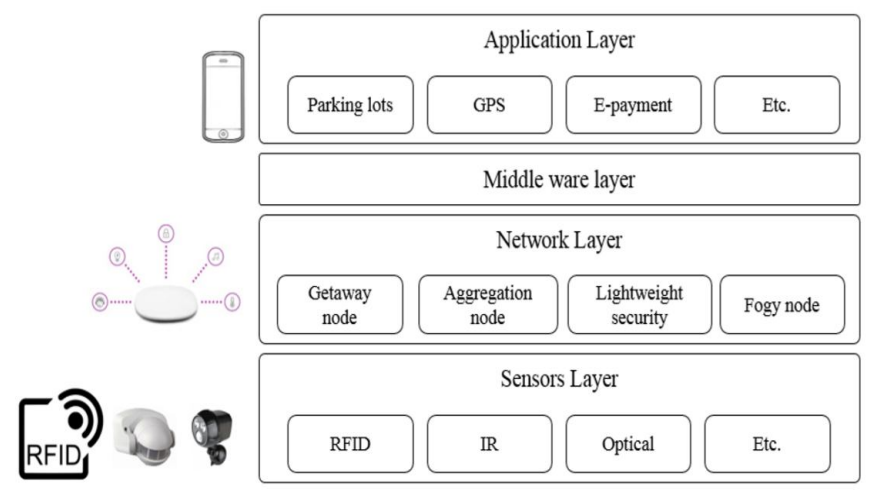

Figure 5: The layers of proposed model

Sensors layer-in every parking area there will be RFID, wireless sensors to detect parking lot status whether it is "occupied" or "free" to increase safety of driver's system adopted emerging technologies such as fog computing, NFC(Near Field Communication). Using such technologies, we can give accurate accident reports which are happening nearby parking location. Figure 6 shows Secure and Smart Parking System model i.e., at the entrance RFID verifies the vehicles whether it is registered or not and the sensors at parking lots intimates that the status of parking lots.

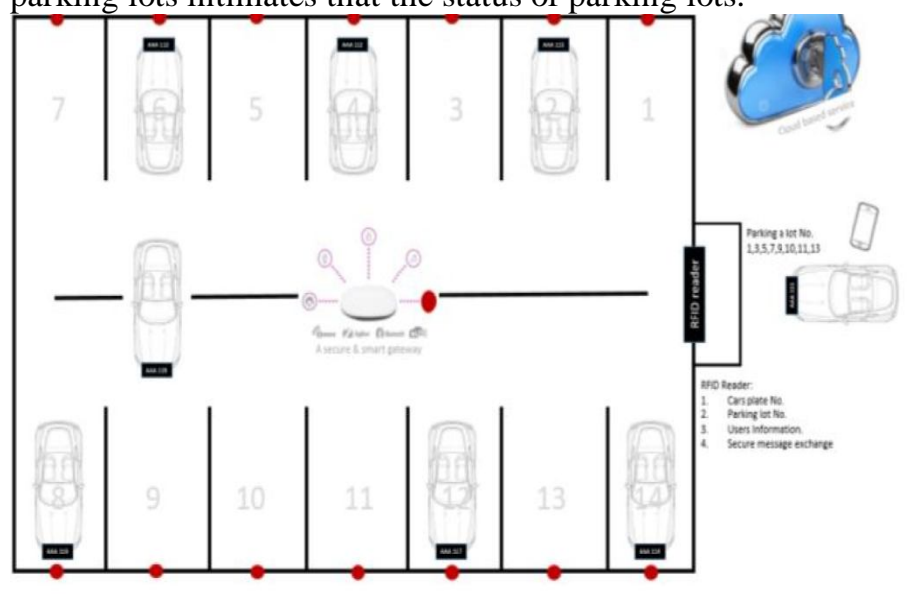

Figure 6: The proposed model of secure and intelligent parking
Ricard Garra proposed system composed of two components RFID (Radio Frequency Identification) and NFC (Near Field Communication) [13]. The NFC on-board interface is activated in this framework, and the app is mounted in a smart phone. This app comes with electronic wallet filled with e-coins. When driver parks the car in the parking lot, the app begins the time span automatically before it exits, then the e-coins are withdrawn from the wallet. This system is better for drivers as it does not require profiles about driver parking habits to be generated and it also avoids double spending (a driver can use more than one parking lot).

Vehicle drivers can download the app and they can request for reserving parking lots at a requested time and requested location as said by Lalit R. Kotecha [14]. Parking service providers will check for availability of parking lots with parking owners, if identified the response is sent to vehicle drivers.

The system consists of three modules.

- user

- parking service

- parking owners

Corresponding parking owners will retrieve the request and reserves the parking lot to that particular vehicle driver and updates the parking lot information. A conformation massage is sent to vehicle driver about reservation of parking lot accordingly, provides parking guidance to navigate to the counts the number of free parking spaces in each of parking lot. RFID costs low for implementing large-scale system. This system reduces the waiting time for parking lots. Vehicle driver using smart phone can reserve the parking lot. The parking lots information is stored in cloud. it will update the data up to date. Using sensors, we can know the allotted parking lot. RFID updates the count of parking lots in real-time. Before entering into parking lot area, it is authenticated via RFID technology (by user card scanning). Unauthorized car cannot enter into parking lot. If the entered information is correct, the driver is allowed to park car. For example, if the parking lots are full, the system will provide information on new parking lot, which includes the address with minimum cost. The new parking lot will be chosen based on neighbor table of current parking lot. ARENA simulation tool is implemented in [].

Joseph Jeffrey describes a system is based on WSN. This system enables car drivers to park indoors and outdoors [15]. Parking spaces are open. The mobile app can be used by vehicle drivers through the installation of an android. The parking lot is identified by sensor node, which costs little and is informed via the Wireless Sensor Network to an embedded Web server. Wi-Fi provides the central web server with the details. So the driver can use your mobile application to find free parking spaces. This system is made up of WSN, EWS, CWS (Central Web Server) and MPA (Smartphone Application). This system includes a computer with a wireless sensor network. Figure 7 shows architecture of smart parking 
system based on WSN. In this smart parking service, the main objectives are

- WSN: It is used to collect all parking lots state (free/occupied) and sends parking lot information to embedded web server.

- EWS: It shall receive data from the wireless sensor networks and send them to CWS (Central Web Server)

- with the parking area location

- CWS: It receives parking lot information from EWS (Embedded Web Server), saves the parking lot information and updates the state of parking lots information in application.

- MPA: It is connected to central web server, it displays the status of parking lots in the nearest parking location, and information is received from central web-server.

Mohammed Y Aalsalem updated the existing parking system by replacing existing sensor or RFID with ANPR (Automatic Number Plate Recognition) cameras for capturing the license number plates of the vehicles [16].

This system, overcomes the following:

- blockage of cars due to wrong parking.

- find out people who are responsible for car damage (car hitting).

- forgetting car park location.

It reduces the parking congestion at parking areas by automatic ticket-free access control systems to provide more efficient enforcement.

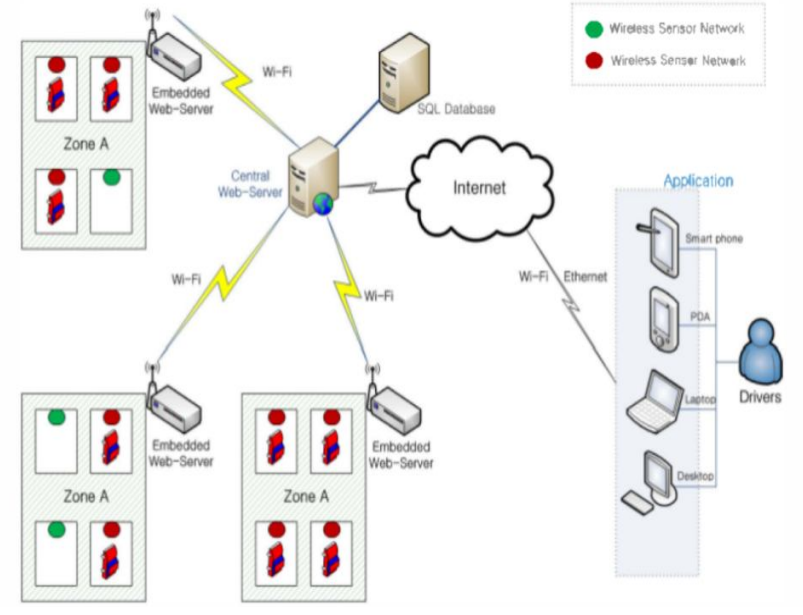

Figure 7: Intelligent Parking Systems design based on wireless sensor networks

Vehicle drivers should install mobile application and register their vehicle with their information to access the location of car where it is parked.

ANPR cameras are mounted at high locations to view whole area. ANPR cameras are located at entry and exit of parking area. ANPR cameras are used to captured the car number plate.

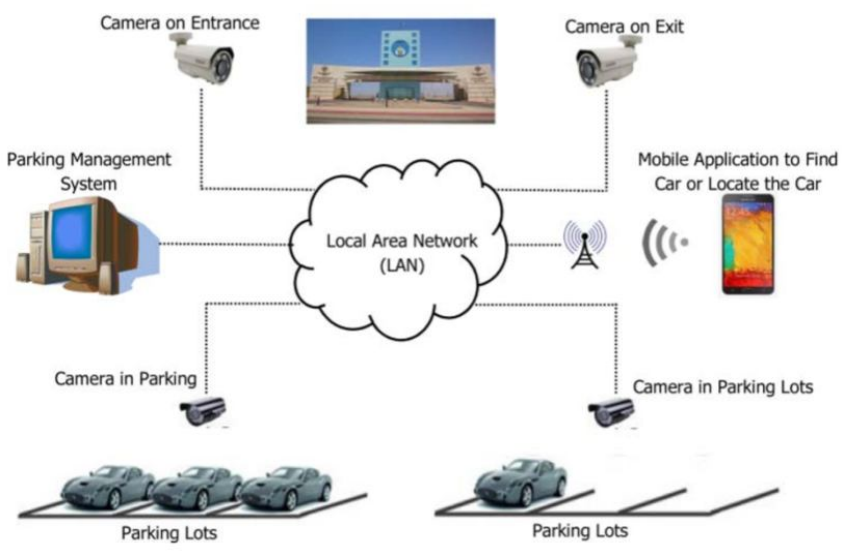

Figure 8: Automated Car Parking Management and Monitoring System (CPMMS) design

Figure 8,demonstrates Design of the Proposed Automated Car Parking Management and Monitoring System, here all the cameras in parking areas and android app are communicated through internet. So, every car that entered and exited information is monitored, by every vehicle is secure from accessing unauthenticated. Information on parking areas and parking lots will already $b$ e stored in the system with other related information (for example, camera is monitoring the parking lot with their physical locations).

Giuseppe Amato et al. explained the detection of vacant parking lots by deep learning [17]. It is a branch of Artificial intelligence that seeks to develop methods that enable computers to learn complicated tasks of perception at the level of human precision, such as seeing and hearing. It offers precise image classification, object detection, speech recognition, processing of natural language, car and pedestrian detection, and more. Deep learning is mainly focus on vision tasks exploits Convolutional Neural Network $(\mathrm{CNN})$. Here based on pictures taken by cameras it updates the status of parking lots. The CNN`s final outputs are the classes for which the network was trained on for a classification issue. Once training is given, the prediction phase is very effective and fast. In this proposed system two different CNN`s training has done and the trained neural networks decides the status of parking lots seen by video cameras. This system is designed with smart cameras i.e., Raspberry Pi 2 model B1, equipped with the standard Raspberry Pi camera module2 which gives clear vision in any light condition and installed in an outdoor camera box on the building`s roof in front of the car parking lots. The parking lots which are nearer to the building are monitored by just one camera, while the farthest from building are observed by more cameras. Two advantages over grounded sensor:

- lower cost

- versatility. here instead of installing one sensor per one parking lot, I can use one smart camera for observing up to 50 parking lots. 
A system based on IoT is said by Ilhan Aydin [18].This system consists of two modules: hardware and software. Hardware consists of parking sensor, gateway node, magnetic sensor. Parking sensor used to get the status of parking lots (whether parking lot ids free /occupied). The parking sensor has 802.15.4 transceiver to transmit status of parking lots information to gateway node (it allows computers within the Local Network to send and receive data over the internet). Gateway node, received information is sent to internet visa GPRS module. A magnetic sensor is used to determine whether the parking lot is free or occupied. Software(the proposed algorithm), Genetic algorithm is used to determine the closest parking lots which are free and to reserve these parking lots to vehicle drivers. Figure 9 illustrates architecture of Cloud-Based Smart-Parking System Based on IoT. Vehicle drivers installs a mobile application (via internet) to find nearest free parking lots. First vehicle drivers have to register for the application. After registration, the vehicle driver current position is tracked by GPS from his mobile to find nearest free parking lots for reservation. Parking lots data and registered user's data is stored in cloud database. Parking sensors sends the information about status of parking lots to the cloud via internet and the same is updated in mobile application for reservation purpose.

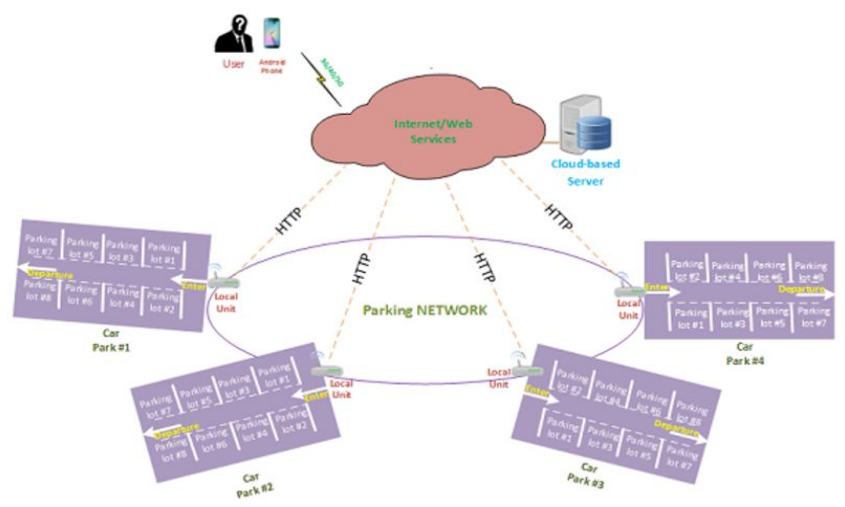

Figure 9: Design of Cloud-Based Smart-Parking System Based on IoT

SecSPS: a secure and privacy-preserved framework for smart parking systems is implemented by Ali Alqazzaz [19]. This proposed system provides real-time parking information for reservation, vehicle park navigation and security from replay attack, man-in-middle, plaintext attacks by using ECC (Elliptic Curve Cryptography) based certificates. Privacy-preserved framework for smart parking systems, by utilizing pub/sub messaging model (here each parking owner is the publisher and each vehicle driver is subscriber. Publisher publishes his own parking lots information and subscriber is the one who is interested in parking lot information. The messages are sent between publisher and subscriber via internet by using transport layer security protocol (TLS). This model guarantees confidentiality, authenticity, integrity.

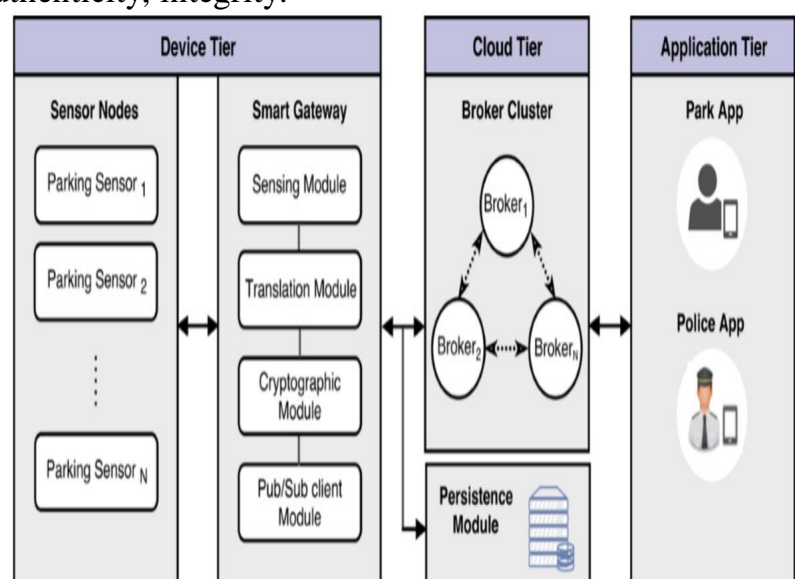

Figure 10: The model of our proposed framework (SecSPS) Figure 10 illustrates the architecture of our proposed framework (SecSPS), i.e., this model consists of three components:

Device tier

- Sensor nodes: Each parking lot is equipped with sensor to give the status of parking lot(free/occupied).

- Smart gateway: Used to receive parking status from sensors, encrypting the information and sent to broker.

\section{Cloud tier}

- Broker: Encrypted messages is received from smart gateway, filtering messages, checks for subscribed clients who are interested for that information and sends that information to subscribed clients. Broken cannot see the messages because they are encrypted.

\section{Application tier}

- Park app: The vehicle driver who all are subscribed for parking lot information.

This proposed system suits for indoor and outdoor deployments (Malls, Airports, and Hospitals).

\section{Working Procedure}

An approved user (admin) initiates a smart contract address transaction to register their vehicle to access the parking area.Once a car arrives at the parking spot, the camera on the parking barrier takes an picture of the license plate and extracts the vehicle registration number (license number) using image recognition techniques.In a smart contract, the vehicle registration number is checked for whether it is registered or not. The barrier will open when registered; otherwise the barrier will remain closed.

\section{CONCLUSION}

Using VANET(vehicle ad-hoc network) the traffic flow information (past data) is collected and divided the data into 
time series (hourly, weekly, day wise) and sent into ANFIS (adoptive neuro-fuzzy system ) to predict traffic flow accurately. With this driver can reserve and arrive the nearest parking lot within less time. As future work, predicting traffic speed(flow) by using online data and past traffic flow data. So, that we can predict density and time taken to each path.The future work is integrating all technologies to achieve most efficient, reliable, inexpensive parking system.

\section{REFERENCES}

1. Wu1, An Empirical Study of Blockchain-based Decentralized Applications arXiv:1902.04969v1 [cs.DC], 13 Feb 2019.

2. Pilkington and Marc,11 Blockchain technology: principles and applications Research handbook on digital transformations, pp. 225, 2016.

3. Bhardwaj, Shweta, and Manish Kaushik, "Blockchain-technology to drive the future Smart Computing and Informatics. Springer, Singapore, Pp. 263-271, 2018.

4. Yan and Gongjun, et al., SmartParking: A secure and intelligent parking system using NOTICE 11th International IEEE Conference on Intelligent Transportation Systems, IEEE, 2008.

5. S. Ahmed, Soaibuzzaman, M. S. Rahman and M. S. Rahaman, A Blockchain-Based Architecture for Integrated Smart Parking Systems IEEE International Conference on Pervasive Computing and Communications Workshops (PerCom Workshops), Kyoto, Japan, pp. 177-182.doi: 10.1109/PERCOMW.2019.8730772, 2019.

6. Rad, Farhad, Hadi Pazhokhzadeh, and Hamid Parvin, A smart hybrid system for parking space reservation in VANET", pp. 11-17, 2017.

7. Amiri, Wesam Al,Mohamed Baza, Karim Banawan, Mohamed Mahmoud, Waleed Alasmary, and Kemal Akkaya, Privacy-Preserving Smart Parking System Using Blockchain and Private Information Retrieval arXiv preprint arXiv:1904.09703, 2019.

8. Faheem, S.A. Mahmud, G.M. Khan, M. Rahman and H. Zafar, A Survey of Intelligent Car Parking System Journal of Applied Research and Technology, Vol. 11, no. 5, pp. 714-726, https://doi.org/10.1016/S1665-6423(13)71580-3, 2013.

9. K. Min and J. Choi, A control system for autonomous vehicle valet parking 13 th International Conference on Control, Automation and Systems (ICCAS 2013), Gwangju, pp. 1714-1717.doi: 10.1109/ICCAS.2013.6704211, 2013.

10. O. Abdulkader, A. M. Bamhdi, V. Thayananthan, K. Jambi and M. Alrasheedi, A novel and secure smart parking management system (SPMS) based on integration of WSN, RFID, and IoT 15th Learning and Technology Conference (L\&T), Jeddah, pp. 102-106, doi: 10.1109/LT.2018.8368492, 2018.
11. Garra, Ricard, Santi Martínez, and Francesc Sebe, A privacy-preserving pay-by-phone parking system IEEE Transactions on Vehicular Technology, 66.7, 2016, pp. 5697-5706.

12. Lalit R. Kotecha, San Ramon, Arda AKSU, Martinez, and Thomas W. Haynes, San Ramon, Mobile Parking Systems and Methods for Providing Real-Time Parking Guidance United States Patent Application Publication,2015, Pub. No.: US 2015/0066545 A1 US 20150.066545A1 KOTECHA.

13. Jeffrey and Joseph, et al. Smart parking system using wireless sensor networks The Sixth International Conference on Sensor Technologies and Applications. 2012.

14. M. Y. Aalsalem, W. Z. Khan and K. M. Dhabbah, An automated vehicle parking monitoring and management system using ANPR cameras 17th International Conference on Advanced Communication Technology (ICACT), Seoul, 2015, pp. 706-710.

15. G. Amato, F. Carrara, F. Falchi, C. Gennaro and C. Vairo, Car parking occupancy detection using smart camera networks and Deep Learning IEEE Symposium on Computers and Communication (ISCC), Messina, 2016, pp. 1212-1217.

16. I. Aydin, M. Karakose and E. Karakose, A navigation and reservation based smart parking platform using genetic optimization for smart cities 2017 5th International Istanbul Smart Grid and Cities Congress and Fair (ICSG), Istanbul, 2017, pp. 120-124.

17. Alqazzaz, Ali, Ibrahim Alrashdi, EsamAloufi, Mohamed Zohdy, and Hua Ming, Secsps: A secure and privacy-preserving framework for smart parking systems Journal of Information Security, pp. 299, 2018.

18. Bhandari, R. R., and Rajasekhar, K. Study on improving the network life time maximazation for wireless sensor network using cross layer approach International Journal of Electrical and Computer Engineering, Vol. 6, no. 6, pp. 3080-3086. doi:10.11591/ijece.v6i6.11208, 2016.

19. Saibaba, C. H. M. H., Uday Kumar, V., Praveenkumar, K., and Bhattacharyya, D. A new privacy approach using graph-EMD International Journal of Software Engineering and its Applications, Vol. 10, no. 7, pp. 11-16, doi:10.14257/ijseia.2016.10.7.02, 2016.

20. Amudhavel, J., Kathavate, P., Reddy, L. S. S., and Satyanarayana, K. V. V. Effects, challenges, opportunities and analysis on security based cloud resource virtualization. Journal of Advanced Research in Dynamical and Control Systems, Vol. 9, Special Issue 12, pp. 1458-1463.

21. Pradhan, A., Sekhar, K. R., and Swain, G. Adaptive PVD steganography using horizontal, vertical, and diagonal edges in six-pixel blocks Security and Communication Networks, doi:10.1155/2017/1924618, 2017.

22. Rao, K. T., and Saidhbi, S. Data security mechanism in private cloud - A case study Journal of Advanced 
Research in Dynamical and Control Systems, Vol. 9, Special Issue 6, pp. 2060-2067, 2017.

23. Rambabu, K., and Venkatram, N. Contemporary affirmation of security and intrusion handling strategies of internet of things in recent literature Journal of Theoretical and Applied Information Technology, Vol. 96, no. 9, pp. 2729-2744, 2018.

24. Shaik, R., and Ahamad, S. S. Security attacks and challenges of wireless sensor network's a review. International Journal of Engineering and Technology(UAE), Vol. 7, 2.32 Special Issue no. 32, pp. 136-138.

25. Sirisha, N., Kiran, K. V. D., and Karthik, R. Hadoop security challenges and its solution using KNOX Indonesian Journal of Electrical Engineering and Computer Science, Vol. 12, no. 1, pp. 107-116. doi:10.11591/ijeecs.v12.i1.pp107-116, 2018.

26. Raghava Rao, K., Sai Sasi Preetham, K., Edukondalu, K., and Ragavendra Reddy, L. V. Connecting vehicles using li-fi technology for reducing accidents. International Journal of Engineering and Technology(UAE), Vol. 7,no. 2, pp. 104-107. doi:10.14419/ijet.v7i2.7.10270, 2018.

27. Anil Kumar, M., Venkata Suman, M., Misra, Y., and Geetha Pratyusha, M. Intelligent vehicle black box using IoT International Journal of Engineering and Technology (UAE), Vol. 7, no. 2, pp. 215-218. doi:10.14419/ijet.v7i2.7.10296, 2018.

28. Raghava Rao, K., Sai Sasi Preetham, K., Edukondalu, K., \& Ragavendra Reddy, L. V. (2018). Connecting vehicles using li-fi technology for reducing accidents. International Journal of Engineering and Technology(UAE), Vol. 7, n0. 2, pp. 104-107, 2018. doi:10.14419/ijet.v7i2.7.10270.

29. Anil Kumar, M., Venkata Suman, M., Misra, Y., \& Geetha Pratyusha, M. (2018). Intelligent vehicle black box using IoT. International Journal of Engineering and Technology (UAE), Vol 7, no. 2, pp. 215-218. doi:10.14419/ijet.v7i2.7.10296.

30. Fitzhan, A., Opportunities and Challenges Blockchain-based Smart Contracts in Indonesia. International Journal of Emerging Trends in EngineeringResearch, Vol. 8, no. 2, pp. 251-258, 2020 doi:10.30534/ijeter/2020/02822020

31. Tatyana Vladimirovna, Deeva Galina Nikiporets-Takigawa, Tatyana Nikolaevna Lustina,

Elena Nikolaevna Podsevalova, Elvira Nikolaevna Didenko, Blockchain Technologies and Smart Contracts: New Technological Methods to Regulate Transactions and Trade Operations, International Journal of Emerging Trends in Engineering Research, Vol 8. No. 7, July 2020 